$\begin{array}{cc}\text { ACADEMIA ROMÂNĂ } & \text { Rev. Roum. Chim., } \\ \text { 2020, 65(3), 283-289 }\end{array}$

\title{
SOLVENT-FREE, MICROWAVE ASSISTED OXIDATION OF ALCOHOLS WITH 4-HYDROXYPYRIDINIUM CHLOROCHROMATE FUNCTIONALIZED SILICA GEL
}

\author{
Shermineh Sadat GHALEHBANDI, Dadkhoda GHAZANFARI, ${ }^{*}$ \\ Sayed Ali AHMADI and Enayatollah SHEIKHHOSSEINI
}

Department of Chemistry, Kerman Branch, Islamic Azad University Kerman, Iran.

Received January 22, 2020

4-Hydroxypyridinium chlorochromate functionalized silica gel was found to be an efficient and reusable oxidant for the very fast oxidation of primary and secondary alcohols to the corresponding carbonyl compounds under solventfree conditions and microwave irradiation in excellent yields.

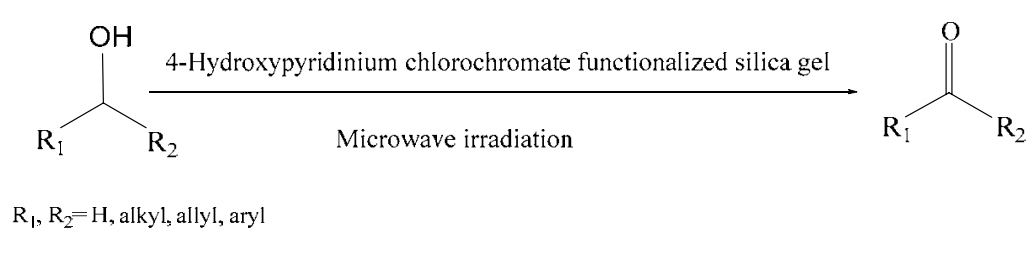

\section{INTRODUCTION}

Oxidation of alcohols to the carbonyl compounds is an important transformation in organic synthesis ${ }^{1}$. Solid supports such as silica gel have found wide application in organic reactions. ${ }^{2-}$ ${ }^{8}$ There are a few example using supported organic molecules on silica gel as oxidizing agents. ${ }^{9-13}$ Since 1986 microwave irradiation has become an increasingly popular method for accelerating synthetic reactions. This technology offers a clean, effective and convenient method of heating, which often results in higher yields, shorter reactions times and easier workup procedure. Organic reactions such as oxidation of alcohols that are assisted by microwave irradiation have attracted considerable attention. ${ }^{14-19}$ Chromium oxidants were used for oxidation of organic compounds consistently. ${ }^{20}$ Pyridinium chlorochromate adsorbed on alumina, ${ }^{21-22}$ on silica, ${ }^{23}$ chromic acid activated carbon, ${ }^{24}$ ammonium chlorochromate on silica, ${ }^{25}$ and chromyl chloride on silica-alumina ${ }^{26}$ have been reported to give better yields under milder conditions as compared to the unsupported oxidants. However, most of these methods have some disadvantages including vigorous reaction conditions and tedious workup, use of solvents and long time of reactions. Thus, there is still a scope for further development of mild, simple and environment-friendly method for oxidation of alcohols to their corresponding carbonyl compounds. During the course of our systematic study on the development of supported reagents and catalysts for the oxidation of organic compounds, ${ }^{27-32}$ we have previously presented a convenient method for oxidation of alcohols to corresponding carbonyl compounds with 4aminopyrinium chlorochromate supported on silica gel. ${ }^{32}$ In this paper we wish to report use of ecofriendly heterogeneous oxidant based on 4hydroxypyridinium chlorochromate functionalized silica gel as an eco-friendly heterogeneous oxidant for the oxidation of alcohols to the corresponding carbonyl compounds.

${ }^{*}$ Corresponding author: dadkhodaghbk@yahoo.com 


\section{RESULTS AND DISSCUSION}

Our approach to a fast and efficient method for the oxidation of alcohols to the corresponding carbonyl compounds is to make use of 4-hydroxypyridinium chlorochromate supported on silica gel (Figure 1). It is a stable solid and can be prepared from the reaction of 4-aminopyridine with activated silica gel, which is then reacted with a solution of chromium trioxide in water.

Initially, the control experiments focused on limiting the quantity of reagent in the mixture, and control of the reaction time. The results are summarized in the Table 1.

As can be seen from Table 1, the best results were obtained with use of Substrate: oxidant ratio (1:1) under 3 min microwave irradiation (entry 10) and additions the irradiation time and the amount of oxidant were unprofitable (entries 2-8, 13). Forde more the reaction was not completed by use of 4-hydroxypyridinium chlorochromate in the absence of silica gel (entry 12). For subsequent experiments on other alkyl, aryl and allyl alcohols, we found that a 1:1 mixture 4-hydroxypyridinium chlorochromate functionalized silica gel and alcohols, was produced the corresponding carbonyl compounds under solvent- free conditions and microwave irradiation (Scheme 1, Table 2). Interestingly, in the oxidation of primary alcohols further oxidation of the liberated aldehydes to the corresponding carboxylic acids didn't occur even with higher mole ratio of 4hydroxypyridinium chlorochromate functionalized silica gel further oxidation didn't perform by reaction time elongation (Table 2 entries 1-24). The chemoselectivity of method also have been investigated using cinnamyl alcohol, in this case the $\mathrm{C}=\mathrm{C}$ stayed intact during the reaction time (Table 2, entries 17-19, 35).
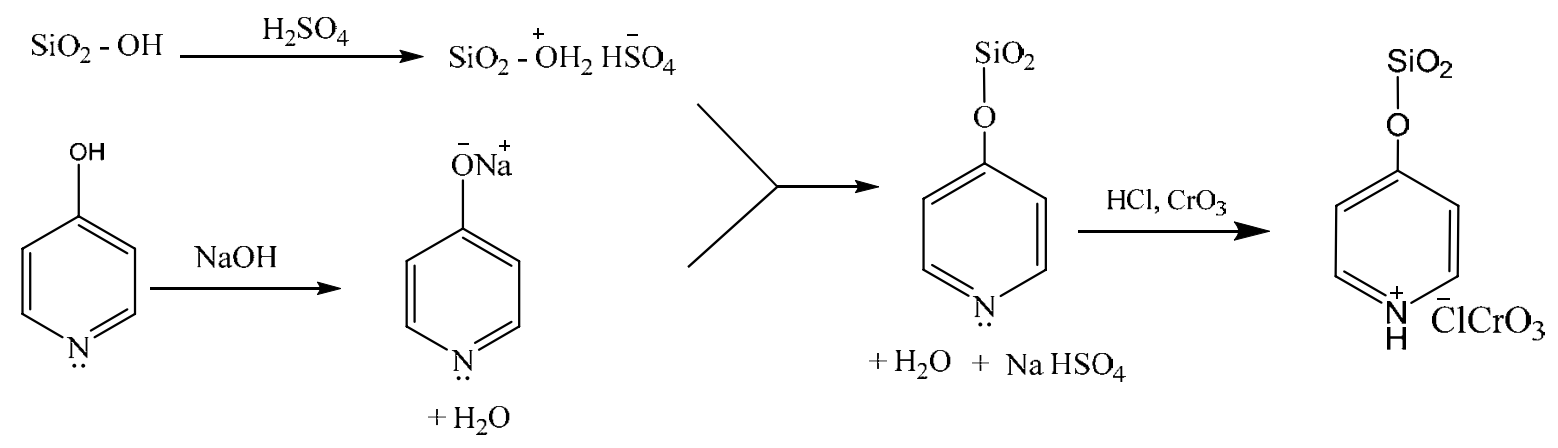

Fig. 1.

Table 1

Optimization of conditions in the reaction of benzyl alcohol with 4-hydroxypyridinium chlorochromate functionalized silica gel

\begin{tabular}{|c|c|c|c|c|c|}
\hline Entry $^{a}$ & $\begin{array}{l}\text { Substrate: } \\
\text { oxidant }^{\mathbf{b}}\end{array}$ & $\begin{array}{l}\text { Irradiation } \\
\text { time(min) }\end{array}$ & Conversion $^{\mathrm{c}}$ & $\begin{array}{l}\text { MW } \\
\text { Power (W) }\end{array}$ & $\begin{array}{l}\text { Yield } \\
(\%)^{d}\end{array}$ \\
\hline 1 & $1: 3$ & $\begin{array}{l}\text { (stirred at } \\
\text { reflux conditions } \\
\text { for } 2 \mathrm{~h} \text { ) }\end{array}$ & 50 & - & 45 \\
\hline 2 & $1: 3$ & 6 & 90 & 300 & 85 \\
\hline 3 & $1: 3$ & 7 & 93 & 300 & 85 \\
\hline 4 & $1: 3$ & 5 & 100 & 400 & 92 \\
\hline 5 & $1: 3$ & 4 & 100 & 400 & 92 \\
\hline 6 & $1: 3$ & 2 & 100 & 400 & 94 \\
\hline 7 & $1: 2$ & 5 & 100 & 400 & 98 \\
\hline 8 & $1: 2$ & 4 & 100 & 400 & 98 \\
\hline 9 & $1: 1$ & 4 & 100 & 400 & 100 \\
\hline 10 & $1: 1$ & 3 & 100 & 400 & 100 \\
\hline 11 & $1: 1$ & 2 & $>97$ & 400 & $>94$ \\
\hline $12 \mathrm{e}$ & $1: 2$ & 8 & $>28$ & 900 & $>20$ \\
\hline 13 & $1: 1$ & 3 & 100 & 500 & 75 \\
\hline
\end{tabular}

${ }^{a}$ Reaction conditions: solvent- free conditions under microwave irradiation.

${ }^{\mathrm{b}}$ Molar ratio.

${ }^{\mathrm{c}}$ Determinated by GC.

${ }^{\mathrm{d}}$ Isolated product.

${ }^{\mathrm{e}}$ In the absence of silica gel. 
<smiles>[R]C([R])O</smiles><smiles></smiles><smiles>[R]C([R2])=O</smiles>

$\mathrm{R}_{1}, \mathrm{R}_{2}=\mathrm{H}$, alkyl, allyl, aryl

Scheme 1 - Oxidation of alcohols with 4-hydroxypyridinium chlorochromate functionalized silica gel.

Table 2

Oxidation of alcohols with 4-hydroxypyridinium chlorochromate functionalized silica gel

\begin{tabular}{|c|c|c|c|c|c|c|}
\hline \multirow{2}{*}{$\begin{array}{l}\text { Ent } \\
\text { ry }\end{array}$} & \multirow[t]{2}{*}{ Substrate } & \multirow[t]{2}{*}{ Product } & \multirow{2}{*}{$\begin{array}{l}\text { Time } \\
(\mathrm{min})\end{array}$} & \multirow{2}{*}{$\begin{array}{l}\text { Yield } \\
(\%)^{a}\end{array}$} & \multicolumn{2}{|c|}{ M. p. $\left({ }^{\circ} \mathrm{C}\right)$} \\
\hline & & & & & Found & $\begin{array}{l}\text { Reporte } \\
\text { d }\end{array}$ \\
\hline 1 & Benzyl alcohol & Benzaldehyde & 2 & 98 & Liq. & \\
\hline 2 & 4-Methylbenzyl alcohol & 4-Methylbenzaldehyde & 2 & 100 & Liq. & \\
\hline 3 & 4-Nitrobenzyl alcohol & 4-Nitrobenzaldehyde & 2 & 98 & $104-105$ & $103-106$ \\
\hline 4 & 4-Methoxybenzyl alcohol & 4-Methoxybenzaldehyde & 2 & 100 & Liq. & \\
\hline 5 & 2,4-Dimethoxybenzyl alcohol & 2,4-Dimethoxybenzaldehyde & 2 & 100 & 70 & $67-69$ \\
\hline 6 & 3-Chlorobenzyl alcohol & 3-Chlorobenzaldehyde & 3 & 98 & Liq. & \\
\hline 7 & 3-Nitrobenzyl alcohol & 3-Nitrobenzaldehyde & 3 & 99 & 56 & $57-58$ \\
\hline 8 & 2-Hydroxybenzyl alcohol & 2-Hydroxybenzaldehyde & 2 & 100 & Liq. & \\
\hline 9 & 3-Hydroxybenzyl alcohol & 3-Hydroxybenzaldehyde & 2 & 99 & 104 & $100-102$ \\
\hline 10 & 2,4-Dichlorobenzyl alcohol & 2,4-Dichlorobenzaldehyde & 3 & 97 & $63-64$ & $64-69$ \\
\hline 11 & 5-Bromo-2-hydroxybenzyl alcohol & 5-Bromo-2-hydroxybenzaldehyde & 3 & 99 & 102 & $100-102$ \\
\hline 12 & $\begin{array}{l}\text { 2-Hydroxy-3-methoxybenzyl } \\
\text { alcohol }\end{array}$ & $\begin{array}{l}\text { 2-Hydroxy-3- } \\
\text { methoxybenzaldehyde }\end{array}$ & 2 & 98 & $41-43$ & $40-42$ \\
\hline 13 & 4-(Dimethylamino)benzyl alcohol & 4-(Dimethylamino)benzaldehyde & 2 & 94 & 77 & $72-75$ \\
\hline 14 & 3,4,5-Trimethoxybenzyl alcohol & 3,4,5-Trimethoxybenzaldehyde & 2 & 97 & 71.5 & $72-74$ \\
\hline 15 & 2-Fluorobenzyl alcohol & 2-Fluorobenzaldehyde & 4 & 96 & Liq. & \\
\hline 16 & 2-Phenylethyl alcohol & Phenylacetaldehyde & 2 & 98 & Liq. & \\
\hline 17 & Cinnamyi alcohol & Cinnamaldehyde & 2 & 91 & Liq. & \\
\hline 18 & 3-Buten-1-ol & Methylviny ketone & 3 & 90 & Liq. & \\
\hline 19 & 1-Pentenol & Pentenal & 4 & 93 & Liq. & \\
\hline 20 & 3-Methyl-1 butanol & 3-Methybutanal & 4 & 94 & Liq. & \\
\hline 21 & Butanol & Butanal & 4 & 87 & Liq. & \\
\hline 22 & 2.2-Dimethyl-1-propanol & 2.2-Dimethylpropanal & 4 & 90 & Liq. & \\
\hline 23 & 1-Hexanol & Hexanal & 4 & 94 & Liq. & \\
\hline 24 & 1-Octanol & Octanal & 4 & 92 & Liq. & \\
\hline 25 & 1-Phenylethyl alcohol & Acetophenone & 2 & 100 & Liq. & \\
\hline 26 & Benzhydrol & Benzophenone & 2 & 100 & $45^{1}$ & $47-51$ \\
\hline 27 & $\begin{array}{l}\text { 9,10-Dihydroxy-9,10- } \\
\text { dihydroanthracene }\end{array}$ & Anthraquinone & 2 & 100 & 280 & $282-285$ \\
\hline 28 & 2,4- Dihydroxypentane & Acetylacetone & 3 & 87 & Liq. & \\
\hline 29 & 4-Chlorobenzhydrol & 4-Chlorobenzophenone & 4 & 91 & 76.5 & $74-76$ \\
\hline 30 & 4-Nitrobenzhydrol & 4-Nitrobenzophenone & 4 & 93 & $133-135$ & $136-138$ \\
\hline 31 & $\begin{array}{l}\text { 2-Amino-9,10-dihydroxy-9,10- } \\
\text { dihydroanthracene }\end{array}$ & 2-Aminoanthraquinone & 4 & 92 & 290 & 291-294 \\
\hline 32 & $\begin{array}{l}\text { 1-Amino-9,10-dihydroxy-9,10- } \\
\text { dihydroanthracene }\end{array}$ & 1-Aminoanthraquinone & 4 & 92 & $250-252$ & $251-254$ \\
\hline 33 & 3-Nitrobenzhydrol & 3-Nitrobenzophenone & 4 & 92 & 81 & $75-78$ \\
\hline 34 & 3-Phenyl-1-propanol & 3-Phenyl-1-propanal & 4 & 88 & Liq. & \\
\hline 35 & 1-Octene-3-ol & 1-Octene-3-one & 4 & 90 & Liq. & \\
\hline 36 & 3-Methyl-2-butanol & 3-Methyl butanone & 4 & 89 & Liq. & \\
\hline 37 & 2-Methyl-1-propanol & 2- Methylpropanal & 4 & 88 & Liq. & \\
\hline 38 & Cyclohexanol & Cyclohexanone & 4 & 91 & Liq. & \\
\hline 39 & 2-Methylcyclopentanol & 2-Methylcyclopentaone & 4 & 91 & Liq. & \\
\hline
\end{tabular}

${ }^{\mathrm{a}}$ Isolated product. 


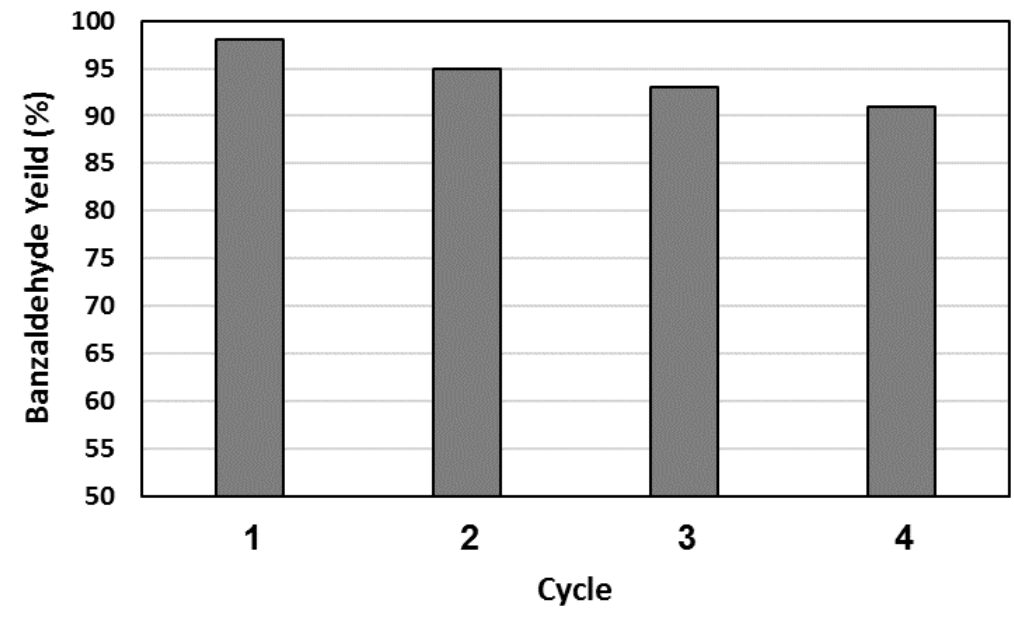

Fig. 2 - Effect of the catalyst recycling on the yield of benzaldehyde for the MW-assisted oxidation of benzyl alcohol with 4-hydroxypyridinium chlorochromate functionalized silica gel.

The reusability of the oxidant has also been investigated. One of the most important features of this heterogeneous can be recycled. 4-Hydroxypyridine functionalized silica gel after reusing for more than four times was recovered quantitatively after each experiment and was activated with a fresh solution of chromium trioxide hydrochloric acid. The activity of the functionalized oxidant was also examined. It was found that the activity decreased gradually, and the obtained benzaldehyde reduced from $100 \%$ to $91 \%$ after four runs (Figure 1). A 1:1 mixture of alcohol /oxidant in a 2 min period was used. The IR spectrum of the recovered reagent exhibited signals at $\mathrm{v}=887$ and $1069 \mathrm{~cm}^{-1}$ corresponding to $\mathrm{Si}-\mathrm{O}-\mathrm{C}$ stretching.

Efficiency and applicability of 4-hydroxypyridinium chlorochromate functionalized silica for the oxidation of alcohols were compared with some of those reported methods. ${ }^{13,16,32-35}$ As shown in Table 3, 4-hydroxypyridinium chlorochromate functionalized silica functions better than previously reported reagents in yields and reaction times for oxidation of primary and secondary alcohols to the corresponding carbonyl compounds under solvent-free conditions and microwave irradiation.

Table 3

Comparison the results of present method with some of those reported in the literature

\begin{tabular}{|c|c|c|c|c|c|c|}
\hline Entry & Substrate & Product & Reaction Conditions & $\begin{array}{l}\text { Yield } \\
(\%) \\
\end{array}$ & Time & Ref. \\
\hline 1 & Benzyl alcohol & Benzaldehyde & $\begin{array}{l}\text { Ratio of } 1: 1.5 \text { alcohol: } 4 \text { - aminopyridinium } \\
\text { chlorochromate }\end{array}$ & 97 & $2(\mathrm{~h})$ & 32 \\
\hline 2 & Benzyl alcohol & Benzaldehyde & $\begin{array}{l}\text { Ratio of } 1: 1 \text { alcohol: SSDT reagent at the } \\
\text { reflux conditions }\end{array}$ & 95 & $1(\mathrm{~h})$ & 13 \\
\hline 3 & Benzyl alcohol & Benzaldehyde & $\mathrm{Rh} / \mathrm{NAC}$ catalysts, $\mathrm{T}=100^{\circ} \mathrm{C}$ & 50 & $24(\mathrm{~h})$ & 33 \\
\hline 4 & Benzyl alcohol & Benzaldehyde & $\begin{array}{l}\text { benzyl alcohol }(1 \mathrm{mmol}), \mathrm{Pd} @ \mathrm{TiC}(25 \mathrm{mg}) \text {, } \\
\mathrm{CH}_{3} \mathrm{CN}(2 \mathrm{~mL}) \text {, } \\
20 \mathrm{~W} \text { domestic bulbs, Air. }\end{array}$ & 97 & $8(h)$ & 35 \\
\hline 5 & Benzyl alcohol & Benzaldehyde & - & 100 & $2(\min )$ & $-{ }^{a}$ \\
\hline 6 & Benzhydrol & Benzophenone & $\begin{array}{l}\text { Ratio of } 1: 1 \text { alcohol: SSDT reagent at the } \\
\text { reflux conditions }\end{array}$ & 83 & $1.5(\mathrm{~h})$ & 13 \\
\hline 7 & Diphenylcarbinol & Benzophenone & -- & 98 & $2(\min )$ & $-{ }^{\mathrm{a}}$ \\
\hline 8 & 1-Hexanol & Hexanal & $\begin{array}{l}\text { Ratio of } 1: 1 \quad \text { alcohol: } \\
\text { Cetyltrimethylammonium Chlorochromate } \\
\text { under microwave irradiation }\end{array}$ & 92 & $8(\min )$ & 16 \\
\hline $\begin{array}{l}9 \\
10 \\
11\end{array}$ & $\begin{array}{l}\text { 1-Hexanol } \\
\text { 1-Phenylethyl alcohol } \\
\text { 1-Phenylethyl alcohol }\end{array}$ & $\begin{array}{l}\text { Hexanal } \\
\text { Acetophenone } \\
\text { Acetophenone }\end{array}$ & Ratio of 1:1: 2 alcohol: $\mathrm{MnO}_{2}$ :TBHP, RT & $\begin{array}{l}94 \\
84 \\
100\end{array}$ & $\begin{array}{l}4(\min ) \\
7(\mathrm{~h}) \\
2(\min )\end{array}$ & $\begin{array}{l}-{ }^{\mathrm{a}} \\
34 \\
-\mathrm{a} \\
34 \\
\end{array}$ \\
\hline
\end{tabular}

\footnotetext{
${ }^{\mathrm{a}}$ Present work
} 


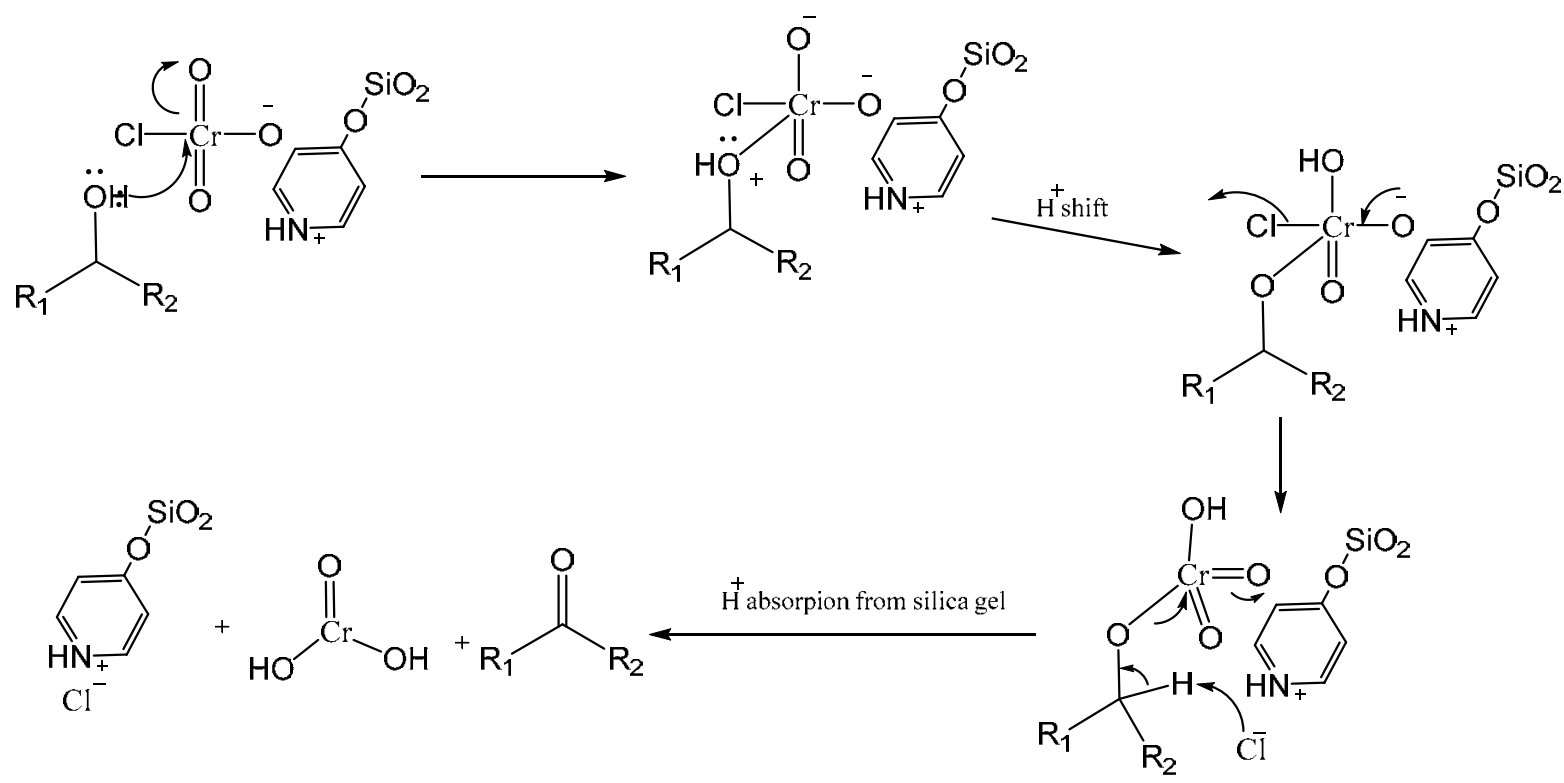

Scheme 2. Suggested mechanism for oxidation of alcohols with 4-hydroxypyridinium chlorochromate functionalized silica gel.

The reaction mechanism depicted in Scheme 2. The first step is attack of oxygen on the chromium to form the Cr-O bond. Then, a proton on the (now positive) $\mathrm{OH}$ is transferred to one of the oxygens of the chromium, and a chloride ion is then displaced. The $\mathrm{C}-\mathrm{O}$ double bond is formed when a base $\left(\mathrm{Cl}^{-}\right)$removes the proton on the carbon adjacent to the oxygen.

\section{EXPERIMENTAL}

\section{Materials and Instruments}

Chemicals were purchased from Merck, Aldrich, Fluka and used without further Purification. Experiments were carried out in closed vessel multi-mode Microsynth Milstone laboratory microwave oven. All experiments had good reproducibility by repeat the experiments in same conditions. The melting points were determined on Electrothermal 9100 apparatus. IR spectra were recorded on a Bruker Tensor 27 spectrometer. ${ }^{1} \mathrm{H}$ NMR and ${ }^{13} \mathrm{C}$ NMR spectra of selected products were recorded on a Bruker $250 \mathrm{MHz}$ spectrometer. To determine substrate conversion, An Agilent 7890A GC was used. All products are known compounds and they were identified by comparison of their physical and spectral data with those of authentic samples. All yields refer to pure isolated products.

\section{Preparation of 4-hydroxypyridine functionalized silica gel}

A fresh solution of $1.9 \mathrm{~g}$ of 4-hydroxypyridine $(20 \mathrm{mmol})$ in $20 \mathrm{~cm}^{3}$ of $\mathrm{NaOH}$ added to $6 \mathrm{~g}$ silica gel (nano powder, $99 \%, 600 \mathrm{~m}^{2} / \mathrm{g}$ ) that was activated by $12.8 \mathrm{~cm}^{3}$ of $4.75 \mathrm{M}$ sulfuric acid and the mixture was stirred at $40{ }^{\circ} \mathrm{C}$ for $10 \mathrm{~min}$ until a milky solid was formed.

\section{Preparation of 4-hydroxypyridinium chlorochromate functionalized silica gel}

To a fresh solution of $2 \mathrm{~g}$ of chromium trioxide $(20 \mathrm{mmol})$ in $25 \mathrm{~mL}$ of $6 \mathrm{~N}$ hydrochloric acid, 4-hydroxypyridine functionalized silica gel (7.9 g, contain $1.9 \mathrm{~g}, 20 \mathrm{mmol} 4-$ hydroxypyridine) was added within 5 min below 7-12 ${ }^{\circ} \mathrm{C}$, and the mixture was stirred at ambient temperature for $30 \mathrm{~min}$ until a lemon solid was formed. After the evaporation of the solvent, the solid was dried at $50{ }^{\circ} \mathrm{C}$ for $2 \mathrm{~h}$. The supported reagent can be kept for weeks in dark without losing its activity. This synthesized reagent $(0.1 \mathrm{~g})$ was titrated by a standard solution of $\mathrm{NaOH}(0.1 \mathrm{~N})$ to obtain its $\left[\mathrm{H}^{+}\right]$ concentration which was 3.6 meq per gram of the powder.

\section{General procedure}

In a small-scale experiment 4-hydroxypyridinium chlorochromate functionalized silica gel $(3.95 \mathrm{~g}$, contain $10 \mathrm{mmol}$ of 4-hydroxypyridinium chlorochromate) an alcohol $(10 \mathrm{mmol})$ was rapidly added at room temperature and the resulting mixture stirred vigorously for the appropriate time. The mixture was irradiated for the time indicated in the table by microwave radiation (Table 2). The progresses of the reactions were monitored by TLC. After cooling to room temperature the product was extracted with diethyl ether $(2 \times 10 \mathrm{~mL})$ and filtered. Evaporation of solvent gave a crude product which was passed through a short silica gel column by ethylacetate: pet. ether (1:7) as eluent to afford the pure product. The structures of the products were confirmed by their melting point, IR and /or NMR spectral data and comparison with commercially available authentic samples. $^{36-38}$

\section{Spectra data}

Benzaldehyde Yield: 100\%; IR (neat) 3064, 3030, 2850, 2821, 2733, 1704, 1655, 1600, 1584, 1456, 1392, 1167, $829 \mathrm{~cm}^{-1}{ }^{1} \mathrm{H}$ NMR $\left(250 \mathrm{MHz}, \mathrm{CDCl}_{3}\right) \delta 9.91(\mathrm{~s}, 1 \mathrm{H}), 7.81-$ $7.89(\mathrm{~m}, 2 \mathrm{H}), 7.42-7.63(\mathrm{~m}, 3 \mathrm{H}) .{ }^{13} \mathrm{C}$ NMR $\left(63 \mathrm{MHz}, \mathrm{CDCl}_{3}\right)$ $\delta 190.91,136.22,134.34,130.12,129.87$.

4-Methoxybenzaldehyde Yield: 98\%. M.p.: 104-105 ${ }^{\circ} \mathrm{C}$.; IR (neat) 2994, 2960, 2852, 1701, 1606, 1538, 1345, 1195, 855 $\mathrm{cm}^{-1} .{ }^{1} \mathrm{H}$ NMR $(250 \mathrm{MHz}, \mathrm{DMSO}) \delta 9.83(\mathrm{~s}, 1 \mathrm{H}), 7.82(\mathrm{~d}$, $2 \mathrm{H}), 7.05(\mathrm{~d}, 2 \mathrm{H}), 3.80(\mathrm{~s}, 3 \mathrm{H}) .{ }^{13} \mathrm{C}$ NMR (63 MHz, DMSO) $\delta$ 191.36, 132.09, 130.08, 114.75, 55.87. 
3-Chlorobenzaldehyde Yield: 98\%; IR (KBr) 3383, 3064, 2938, 2833, 2699, 1696, 1474, 1388, 1195, $790 \mathrm{~cm}^{-1}{ }^{1} \mathrm{H}$ NMR $\left(250 \mathrm{MHz}, \mathrm{CDCl}_{3}\right) \delta 9.98(\mathrm{~s}, 1 \mathrm{H}), 7.43-7.78(\mathrm{~m}, 3 \mathrm{H}) \cdot{ }^{13} \mathrm{C}$ NMR $\left(63 \mathrm{MH} z, \mathrm{CDCl}_{3}\right) \delta 191.3,138.22,136.17,134.09$, $131.23,130.02,126.74$.

2-Hydroxybenzaldehyde Yield: 100 \%; IR (neat) 2994, 2960, $2852,1701,1606,1538,1345,1195,855 \mathrm{~cm}^{-1}$ 13C NMR (63 $\mathrm{MH} z$, DMSO) $\delta 197.03,162.53,136.29,132.86,121.45$, $120.37,116.71$

2-Hydroxy-3-methoxybenzaldehyd: Yield: 98 \%. M.p.: 41-43 ${ }^{\circ} \mathrm{C} . ;$ IR (KBr) 3448, 1640, 1587, 1454, $1255 \mathrm{~cm}^{-1}{ }^{1} \mathrm{H}$ NMR $\left(250 \mathrm{MHz}, \mathrm{CDCl}_{3}\right) 10.913(\mathrm{~s}, 1 \mathrm{H}), 9.72(\mathrm{~s}, 1 \mathrm{H}), 6.76-6.99$ (m, 4H), 3.67(s, 3H). ${ }^{13} \mathrm{C}$ NMR $\left(63 \mathrm{MHz}, \mathrm{CDCl}_{3}\right) \delta 196.54$, $151.33,148.02,124.32,120.63,119.46,117.82,56.02$.

4-(Dimethylamino)benzaldehyde Yield: 94 \%. M.p.: $77{ }^{\circ} \mathrm{C}$.; IR (KBr) 3447, 2923, 2714, 1661, 1596, 1547, 1359, 1162 , $812 \mathrm{~cm}^{-1}{ }^{1} \mathrm{H}$ NMR $(250 \mathrm{MHz}$, DMSO) $\delta 9.81(\mathrm{~s}, 1 \mathrm{H}), 7.84(\mathrm{~d}$, $2 \mathrm{H}), 6.73(\mathrm{~d}, 2 \mathrm{H}), 2.98(\mathrm{~s}, 6 \mathrm{H}) .{ }^{13} \mathrm{C}$ NMR $(63 \mathrm{MHz}$, DMSO) $\delta$ 191.13, 155.43, 132.67, 126.20. 111.36, 40.17.

2-Fluorobenzaldehyd Yield: $96 \%$; IR (neat) 3448, 2827, 2738, 1699, 1598, 1296, $833 \mathrm{~cm}^{-1}{ }^{1} \mathrm{H}$ NMR $(250 \mathrm{MHz}$, $\left.\mathrm{CDCl}_{3}\right) \delta 10.16(\mathrm{~d}, 1 \mathrm{H}), 7.56(\mathrm{~d}, 1 \mathrm{H}), 7.33(\mathrm{dd}, 1 \mathrm{H}), 7.15(\mathrm{dd}$, $1 \mathrm{H}), 7.46 .984(\mathrm{~d}, 1 \mathrm{H}) .{ }^{13} \mathrm{C} \mathrm{NMR}\left(63 \mathrm{MHz}, \mathrm{CDCl}_{3}\right) \delta 185.93$, $158.19,135.57,129.19,123.77,122.92,115.83$.

Cinnamaldehyde Yield: $91 \%$; IR (neat) 3063, 2993, 2816, $1678,1123,975,748 \mathrm{~cm}^{-1}{ }^{1} \mathrm{H}$ NMR $\left(250 \mathrm{MHz}, \mathrm{CDCl}_{3}\right) \delta 9.58$ $(\mathrm{d}, 1 \mathrm{H}), 7.41-7.57(5 \mathrm{H}), 7.51(\mathrm{~d}, 1 \mathrm{H}), 6.72(\mathrm{~d}, 1 \mathrm{H}) .{ }^{13} \mathrm{C}$ NMR $\left(63 \mathrm{MH} z, \mathrm{CDCl}_{3}\right) \delta 192.61,151.95,133.88,130.84,128.21$, 127.76, 127.19.

Octanal: Yield: $92 \%$; IR (neat) 2960, 2874, 1730, $1411 \mathrm{~cm}^{-1}$ ${ }^{1} \mathrm{H}$ NMR $\left(250 \mathrm{MHz}, \mathrm{CDCl}_{3}\right) \delta 9.68(\mathrm{t}, 1 \mathrm{H}), 2.33(\mathrm{~m}, 2 \mathrm{H}), 1.60$ (m, 2H), 1.25-1.29 (m, 8H), 0.82 (t, 3H). ${ }^{13} \mathrm{C}$ NMR $(63 \mathrm{MHz}$, $\left.\mathrm{CDCl}_{3}\right) \delta 192.47,43.13,31.05,30.10,29.24,23.37$.

Acetophenone Yield: 100 \%; IR (neat) 3080, 3025, 1696, $1605,1365,1268,762 \mathrm{~cm}^{-1},{ }^{1} \mathrm{H}$ NMR $\left(250 \mathrm{MHz}, \mathrm{CDCl}_{3}\right) \delta$ 7.57-7.83 (m, 5H), 2.47(s, 3H). ${ }^{13} \mathrm{C}$ NMR $\left(63 \mathrm{MHz}, \mathrm{CDCl}_{3}\right) \delta$ $190.78,135.94,129.92,129.14,128.96,25.88$.

Acetylacetone Yield: 87 \%.; IR (neat) 3443, 2360, 1716, 1623 $\mathrm{cm}^{-1}{ }^{1} \mathrm{H}$ NMR (250 MHz, DMSO) $\delta 3.59(\mathrm{~s}, 2 \mathrm{H}), 1.989(\mathrm{~s}$, $6 \mathrm{H}),\left[15.4(\mathrm{O}-\mathrm{H}), 5.6\right.$ (vinyl $\mathrm{H}$ in enol form)]. ${ }^{13} \mathrm{C} \mathrm{NMR}$ $(63 \mathrm{MH} z, \mathrm{DMSO}) \delta 203.13,57.98,30.55,[191.46,100.51$, 29.50, 24.43, in enol form].

4-Chlorobenzophenone Yield: 91 \%. M.p.: $76.5{ }^{\circ} \mathrm{C} . ;{ }^{1} \mathrm{H}$ NMR $\left(250 \mathrm{MHz}, \mathrm{CDCl}_{3}\right) \delta 7.82(\mathrm{~d}, 2 \mathrm{H}), 7.63(\mathrm{~d}, 2 \mathrm{H}), 7.53(\mathrm{dd}, 1 \mathrm{H})$, $7.42(\mathrm{dd}, 2 \mathrm{H}), 7.39(\mathrm{~d}, 2 \mathrm{H}) .{ }^{13} \mathrm{C} \mathrm{NMR}\left(63 \mathrm{MHz}, \mathrm{CDCl}_{3}\right) \delta$ $194.86,137.82,136.64,136.02,131.91,130.63,128.85$, $128.12,127.50$.

4-Nitrobenzophenone Yield: 93 \%. M.p.: 133-135 ${ }^{\circ} \mathrm{C}$; IR (KBr) 3382, 2751, 1666, 1278, $763 \mathrm{~cm}^{-1}{ }^{-1} \mathrm{H}$ NMR $(250 \mathrm{MHz}$, $\left.\mathrm{CDCl}_{3}\right) \delta 8.17(\mathrm{~d}, 2 \mathrm{H}), 7.86(\mathrm{~d}, 2 \mathrm{H}), 7.75(\mathrm{~d}, 2 \mathrm{H}), 7.53(\mathrm{~m}$, $1 \mathrm{H}), 7.44(\mathrm{~m}, 2 \mathrm{H}) .{ }^{13} \mathrm{C} \mathrm{NMR}\left(63 \mathrm{MHz}, \mathrm{CDCl}_{3}\right) \delta 194.15$, 149.91. 142.86, 137.25, 130.41, 128.87, 127.53, 126.27, 123.46,

2-Aminoanthraquinone Yield: $92 \%$. M.p.: $290^{\circ} \mathrm{C}$.; IR (KBr) $3447,1587,1454,1256,1215,1161,1058,946,761 \mathrm{~cm}^{-1} .{ }^{1} \mathrm{H}$ NMR $\left(250 \mathrm{MH} z\right.$, DMSO) $\delta 6.62-8.04(8 \mathrm{H}), 2.46(2 \mathrm{H}) .{ }^{13} \mathrm{C}$ NMR $(63 \mathrm{MH} z, \mathrm{DMSO}) \delta 183.72,180.49,155.09,135.27$, $134.62,134.05,129.95,126.73,121.58,118.49,110.10$.
1-Aminoanthraquinone Yield: $92 \%$. M.p.: $250-252{ }^{\circ} \mathrm{C}$.; IR (KBr) 3419, 2359, 1637, 1607, 1283, 1013, $801 \mathrm{~cm}^{-1}{ }^{1} \mathrm{H}$ NMR $(250 \mathrm{MHz}, \mathrm{DMSO}) \delta 8.12(\mathrm{~m}, 1 \mathrm{H}), 8.03(\mathrm{~d}, 1 \mathrm{H}), 7.66(\mathrm{~m}, 1 \mathrm{H})$, 7.41(m, 1H), 7.38(d, 1H), $7.22(\mathrm{~m}, 1 \mathrm{H}), 7.06(\mathrm{~d}, 1 \mathrm{H}), 2.78(\mathrm{~s}$, 2H). ${ }^{13} \mathrm{C}$ NMR (63 MHz, DMSO) $\delta 183.30,182.91,153.11$, $135.51,134.70,133.28,132.09,131.81,131.03,127.41$, 124.68, 122.27, 116.37, 110.89 .

2- Methylpropanal Yield: $88 \%$; IR (neat) 2742, 1727, 1120, $933 \mathrm{~cm}^{-1}{ }^{1} \mathrm{H}$ NMR $\left(250 \mathrm{MHz}, \mathrm{CDCl}_{3}\right) \delta 9.74(\mathrm{~d}, 1 \mathrm{H}), 2.77(\mathrm{~m}$, $1 \mathrm{H}), 1.15(\mathrm{~d}, 6 \mathrm{H}) .{ }^{13} \mathrm{C} \mathrm{NMR}\left(63 \mathrm{MH} z, \mathrm{CDCl}_{3}\right) \delta 203.87$, $42.01,16.76$.

2-Methylcyclopentaone Yield: $91 \%$; IR (neat) 3473, 2971, 2927, 1745, 1453, 1228, 1156, $898 \mathrm{~cm} \cdot{ }^{-1}{ }^{1} \mathrm{H}$ NMR $(250 \mathrm{MHz}$, $\left.\mathrm{CDCl}_{3}\right) 2.40(\mathrm{~m}, 1 \mathrm{H}), 2.28(\mathrm{~m}, 1 \mathrm{H}), 2.17(\mathrm{~m}, 1 \mathrm{H}), 1.96(\mathrm{~m}$, $1 \mathrm{H}), 1.75(\mathrm{~m}, 1 \mathrm{H}), 1.51(\mathrm{~m}, 1 \mathrm{H}), 1.13(\mathrm{~m}, 1 \mathrm{H}) .{ }^{13} \mathrm{C} \mathrm{NMR}(63$ $\left.\mathrm{MHz}, \mathrm{CDCl}_{3}\right) \delta 222.35,44.28,38.16,32.84,21.07,15.26$.

\section{CONCLUSION}

In conclusion, we have designed to synthesize 4-hydroxypyridinium chlorochromate functionalized silica as an efficient and recoverable oxidant for controlled oxidation of alcohols to their corresponding carbonyl compounds. Efficiency, high yields, short reaction times, clean reaction profile, solvent-free condition, simplicity of preparation and recyclability of oxidant are some advantages of the described protocol.

Acknowledgements: Islamic Azad University is strongly appreciated for its financial support to our research group.

\section{REFERENCES}

1. B. M. Trost, "Comprehensive Organic Synthesis, Pergamum“, New York, 1997.

2. P. Laszlo, "Preparative Chemistry Using Supported Reagents“, Academic Press, Sn. C. A. Diego, 1987.

3. K. Smith, "Solid Supports and Catalyst in Organic Synthesis", Ellis Harwood, Chi Chester, 1992.

4. N. K. Terrett, "Combinatorial Chemistry", Oxford University Press, New York, 1998.

5. a). M. C. Killop and D. W. Young, Synthesis, 1979, 401408; b) A. M. C. Killop, D. W. Young, Synthesis, 1979, 481-500.

6. H. Alshammari, M. Alhumaimess and M. H. Alotaibi, $J$. King Saud Univ.-Scie., 2017, 29, 561-566.

7. H. Ghafuri, Current Chem. Lett., 2015, 4, 27-32.

8. A. Aminimanesh and T. Nazari, J. Chil . Chem. Soc., 2015, 60, 3001-3004.

9. M. H. Ali, G. J. Bohnert, Synthesis, 1998, 1238-1240.

10. S. Borkar, D. Khadikab, M. Bhushan, Synth Commun., 1999, 29, 4295-4298.

11. C. Bolm, Chem. Commun., 1999, 18, 5057-5065.

12. M. M. Hashemi, A. Rahimi, Z. Karim and Z. Jaberi, Acta. Chim. Slov., 2005, 52, 86-87.

13. F. M. Matloubi, N. Masoud and B. K. Foroushani, Scientia Iranica, 2013, 20, 598-602. 
14. J. C. Lee, J. Y. Lee and S. J. Lee, Tetrahedron Lett., 2004, 45, 4939-4941.

15. D. Bogdal and M. Luksasiewicz, Synlet, 2000, 1, 143145.

16. M. K. Mohammadi, S. Ghammamy and H. Imanieh, Chines J. Chem., 2009, 27, 1501-1504.

17. I. S. Cozzi, C. Crotti and E. Farnetti, J. Organometallic Chem., 2018, 878, 38-47.

18. M. K. Mohammadi, Open J. Syn. Theory Appl., 2013, 2, 87-90.

19. J. C. Lee, J. Y. Lee and J. M. Lee, Bull. Korean Chem. Soc., 2005, 26, 1300-1302.

20. B. Maurer and A. Hauser, Tertrahedron Lett., 1984, 25, 1061-1062.

21. a) S. Bhar and S. C. Kumar, Tetrahedron, 2003, 59, 34933498. b) R. P. Sengh, H. N. Subbaro and S. Dev, Tertrahedron, 1979, 20, 1789-1793.

22. M. M. Heravi, R. Kiakoojori and M. M. Aghayan, Monatsh. Chem., 1999, 130, 481-483.

23. F. A. Fitch, W. J. Moore and K. J. Mudd, J. Chem. Edu., 1999, 76, 974975.

24. A. J. Alamdany and T. W. Jihad, Tiktit J. Pure Sci., 2012, 17, 72-76.

25. M. M. Heravi, R. Kiakoojori and M. M. Mojtahedi, Indian J. Chem. B, 2001, 40, 329-330.

26. J. S. F. Jr and C. I. C. Chern, J. Org. Chem., 1977, 42, 2182-2183.
27. M. M. Hashemi and Y. A. Beni, Iran J. Chem. \& Chem. Eng., 1998, 9, 238-239.

28. M. M. Hashemi, D. Ghazanfari and Z. Karimi-Jaberi, Monatsh. Chem., 2004, 135, 185-188.

29. M. M. Hashemi, Z. Karimi-Jaberi and D. Ghazanfari, J. Chem. Res., 2004, 364-365.

30. D. Ghazanfari, F. Najafizadeh and F. Khosravi, Monatsh. Chem., 2004, 135, 1409-1413.

31. M. M. Hashemi, D. Ghazanfari, Y. Ahmadibeni, Z. Karimi-Jaberi and A. Ezabadi, Synthesis Commun., 2005, 35, 1103-1107.

32. M. M. Hashemi, D. Ghazanfari and M. Akhbari, Monatsh. Chem., 2004, 135, 739-743.

33. A. W. Coner, R. Yulia, M. F. Ian, P. Liam, J. Raliton, M. Bravo-Sanchez, N. Sano, P. J. Cumpson, P. D. Coates, X. Liu and M. Conte, Appl. Catalysis A, General, 2019, 570, 271-282.

34. C. Bhaumik, D. Stein, S. Vincendeau, R. Poli and E. Manoury, Comptes Rendus Chimie, 2016, 19, 566-570.

35. S. Verma, N. R. B. Baig, M. N. Nadagouda and R. S. Varma, Tetrahedron, 2017, 73, 5577-5580.

36. A. Aldrich, "Handbook of Fine Chemicals and Laboratory Equipment's“, 2008.

37. C. J. Pouchert, "The Aldrich Library of IR Spectra", Aldrich Chemical Co, Milwaukee, 1981.

38. C. J. Pouchert, "The Aldrich Library of NMR Spectra“, Aldrich Chemical Co, Milwaukee, 1983. 
